\title{
Restricted sums in a field
}

\author{
by \\ QING-Hu Hou (Tianjin) and ZHI-Wei Sun (Nanjing)
}

1. Introduction. Let $\mathbb{Z}_{p}=\mathbb{Z} / p \mathbb{Z}$ stand for the field of all residue classes modulo prime $p$. In 1964 P. Erdős and H. Heilbronn (cf. [EH] and $[\mathrm{Gu}]$ ) conjectured that for each nonempty subset $A$ of $\mathbb{Z}_{p}$ there are at least $\min \{p, 2|A|-3\}$ residue classes modulo $p$ that can be written as the sum of two distinct elements of $A$. This had been open for thirty years until J. A. Dias da Silva and Y. O. Hamidoune $[\mathrm{DH}]$ proved the following result with the help of the representation theory of symmetric groups.

The Dias da Silva-Hamidoune Theorem. Let $F$ be any field and $n$ a positive integer. Then for any finite subset $A$ of $F$ we have

$$
\left|n^{\wedge} A\right| \geq \min \left\{p(F), n|A|-n^{2}+1\right\},
$$

where $n^{\wedge} A$ denotes the set of all sums of $n$ distinct elements of $A$, and $p(F)$ represents the additive order of the multiplicative identity of $F$.

Let $F$ be a field and $e$ be its multiplicative identity. If $e$ has a finite order as an element of the additive group of $F$, then the order $p(F)$ is a prime and is called the characteristic of $F$; otherwise, $p(F)$ is $+\infty$ and the characteristic of $F$ is usually said to be 0 .

In 1995-1996, N. Alon, M. B. Nathanson and I. Z. Ruzsa [ANR1, ANR2] invented a polynomial method to obtain results similar to the Dias da SilvaHamidoune theorem.

By means of the polynomial method and the determination of certain coefficients in a polynomial in product form, we obtain

THEOREM 1.1. Let $k, m$ be nonnegative integers and $n$ a positive integer. Let $F$ be a field of characteristic $p$ where $p$ is zero or a prime with $p / n$ greater than $m$ and $k+m-m n-1$. Let $A_{1}, \ldots, A_{n}$ be subsets of $F$ with

2000 Mathematics Subject Classification: Primary 11B75; Secondary 05A05, 11C08.

The second author is responsible for all the communications, and supported by the Teaching and Research Award Program for Outstanding Young Teachers in Higher Education Institutions of MOE, and the National Natural Science Foundation of P.R. China. 
cardinality $k$. For any $i, j=1, \ldots, n$ with $i \neq j$, let $S_{i j} \subseteq F$ and $\left|S_{i j}\right| \leq m$. Then, for the set

$$
C=\left\{a_{1}+\ldots+a_{n}: a_{1} \in A_{1}, \ldots, a_{n} \in A_{n}, a_{i}-a_{j} \notin S_{i j} \text { if } i \neq j\right\},
$$

we have

$$
|C| \geq(k+m-m n-1) n+1 .
$$

REMARK 1.1. In the case $m=0$, the result also follows from the well known Cauchy-Davenport theorem (cf. Theorem 2.2 of $[\mathrm{N}]$ ) which asserts that for any finite nonempty subsets $A$ and $B$ of a field $F$ we have $|A+B| \geq$ $\min \{p(F),|A|+|B|-1\}$. When $m=1$ and $S_{i j}=\{0\}$, the set $C$ given by (1.2) coincides with $n^{\wedge} A$ if $A_{1}=\ldots=A_{n}=A$. Since $(k+m-m n-1) n-(k-1)=$ $(k-1-m n)(n-1)$, the condition $p(F)>n \max \{m, k+m-m n-1\}$ implies that $k \leq p(F)$. If the condition $p(F)>(k+m-m n-1) n$ in Theorem 1.1 is violated, then $k^{\prime}+m-m n-1=[(p(F)-1) / n]$ for some $0<k^{\prime}<k$ (where $[\alpha]$ denotes the greatest integer not exceeding the real number $\alpha$ ), thus for a certain $C^{\prime} \subseteq C$ we have

$$
|C| \geq\left|C^{\prime}\right| \geq\left(k^{\prime}+m-m n-1\right) n+1=n\left[\frac{p(F)-1}{n}\right]+1 .
$$

For convenience we now set

$$
\mathbb{N}=\{0,1,2, \ldots\} \quad \text { and } \quad \mathbb{Z}^{+}=\{1,2,3, \ldots\} .
$$

If $k, l \in \mathbb{Z}$ then we put

$$
[k, l)=\{x \in \mathbb{Z}: k \leq x<l\} \quad \text { and } \quad[k, l]=\{x \in \mathbb{Z}: k \leq x \leq l\} .
$$

The following example shows that the lower bound in (1.3) can be attained if it is positive.

EXAMPLE 1.1. Let $F$ be a field and $e$ be its multiplicative identity. Let $k, m \in \mathbb{N}, n \in \mathbb{Z}^{+}$and $m(n-1)<k \leq p(F)$. Set $A_{1}=\ldots=A_{n}=\{x e: x \in$ $[0, k)\}, S=\{x e: x \in[0, m)\}$ and

$$
C=\left\{a_{1}+\ldots+a_{n}: a_{1} \in A_{1}, \ldots, a_{n} \in A_{n}, a_{i}-a_{j} \notin S \text { if } i \neq j\right\} .
$$

Then $\left|A_{1}\right|=\ldots=\left|A_{n}\right|=k,|S| \leq m$ and $C=\{x e: x \in I\}$ where

$$
I=\left\{a_{1}+\ldots+a_{k}: a_{1}, \ldots, a_{k} \in[0, k),\left|a_{i}-a_{j}\right| \geq m \text { whenever } i \neq j\right\} .
$$

Observe that $I$ is the union of the following intervals:

$$
\begin{aligned}
& 0+m+2 m+\ldots+(n-3) m+(n-2) m+[(n-1) m, k-1], \\
& 0+m+2 m+\ldots+(n-3) m+[(n-2) m, k-1-m]+k-1, \\
& {[0, k-1-(n-1) m]+(k-1-(n-2) m)+\ldots+(k-1-m)+(k-1) .}
\end{aligned}
$$


Therefore

$$
I=\left[\sum_{r=0}^{n-1} r m, \sum_{r=0}^{n-1}(k-1-r m)\right]=\left[\frac{m n(n-1)}{2},(k-1) n-\frac{m n(n-1)}{2}\right]
$$

and $|I|=(k+m-m n-1) n+1$. So $|C|=\min \{p(F),(k+m-m n-1) n+1\}$.

Corollary 1.1. Let $k \in \mathbb{N}, m, n \in \mathbb{Z}^{+}$and $k>m(n-1)$. Let $F$ be a field with $p(F)>n \max \{m, k-1-m(n-1)\}$, and $A_{1}, \ldots, A_{n}$ be subsets of $F$ with cardinality $k$. Let $b_{1}, \ldots, b_{n} \in F, 0 \in S \subseteq F$ and $|S|=m$. Then the set

$$
\left\{a_{1}+\ldots+a_{n}: a_{i} \in A_{i}, a_{i} \neq a_{j} \text { and } a_{i}+b_{i}-\left(a_{j}+b_{j}\right) \notin S \text { if } i \neq j\right\}
$$

is nonempty, and its cardinality is greater than $(k-1-m(n-1)) n$.

Proof. For $1 \leq i<j \leq n$ we put

$$
S_{i j}=\{0\} \cup\left\{x-b_{i}+b_{j}: x \in S \backslash\{0\}\right\} \quad \text { and } \quad S_{j i}=\left\{x-b_{j}+b_{i}: x \in S\right\} .
$$

Applying Theorem 1.1 we immediately get the required result.

REMARK 1.2. The fact that (1.4) is nonempty under the assumptions of Corollary 1.1 was realized by Alon [A2] in the case $F=\mathbb{Z}_{p}$ with $p$ being a prime. In the special case $k=n, m=1$ and $S=\{0\}$, the result implies that for any odd prime $p$ and subsets $A, B$ of $\mathbb{Z}_{p}$ with cardinality $n$, there is a numbering $\left\{a_{i}\right\}_{i=1}^{n}$ of the elements of $A$ and a numbering $\left\{b_{i}\right\}_{i=1}^{n}$ of those in $B$ such that the sums $a_{1}+b_{1}, \ldots, a_{n}+b_{n}$ are distinct. In fact, H. S. Snevily [Sn] even conjectured that the above $\mathbb{Z}_{p}$ can be replaced by any abelian group whose order is odd.

Let us end this section with a conjecture posed by the second author.

Conjecture 1.1. Let $F$ be any field, and $A_{1}, \ldots, A_{n}$ be subsets of $F$ which are finite and nonempty. For $1 \leq i<j \leq n$ let $S_{i j}$ and $S_{j i}$ be finite subsets of $F$ with $\left|S_{i j}\right| \equiv\left|S_{j i}\right|(\bmod 2)$. Then, for the set $C$ given by (1.2), we have

$$
|C| \geq \min \left\{p(F), \sum_{i=1}^{n}\left|A_{i}\right|-\sum_{1 \leq i<j \leq n}\left(\left|S_{i j}\right|+\left|S_{j i}\right|\right)-n+1\right\} .
$$

The conjecture is open even when $F$ is the rational field $\mathbb{Q}$; the reader may consult $[\mathrm{Su}]$ for related results.

\section{Two auxiliary propositions}

Proposition 2.1. Let $A_{1}, \ldots, A_{n}$ be finite subsets of a field $F$ with $\left|A_{i}\right| \geq k_{i}$ for $i \in[1, n]$ where $k_{1}, \ldots, k_{n} \in \mathbb{Z}^{+}$. Let $\lambda\left(x_{1}, \ldots, x_{n}\right), \mu\left(x_{1}, \ldots, x_{n}\right)$ $\in F\left[x_{1}, \ldots, x_{n}\right]$ and $\operatorname{deg} \mu>0$. Put

$$
C=\left\{\mu\left(a_{1}, \ldots, a_{n}\right): a_{1} \in A_{1}, \ldots, a_{n} \in A_{n}, \lambda\left(a_{1}, \ldots, a_{n}\right) \neq 0\right\} .
$$


Then there is no $\omega\left(x_{1}, \ldots, x_{n}\right) \in F\left[x_{1}, \ldots, x_{n}\right]$ such that

$$
\lambda\left(x_{1}, \ldots, x_{n}\right) \omega\left(x_{1}, \ldots, x_{n}\right) \mu\left(x_{1}, \ldots, x_{n}\right)^{|C|}
$$

is of degree $\sum_{i=1}^{n}\left(k_{i}-1\right)$ and the coefficient of $x_{1}^{k_{1}-1} \ldots x_{n}^{k_{n}-1}$ is nonzero.

Proof. Suppose that such an $\omega\left(x_{1}, \ldots, x_{n}\right)$ exists. Write

$$
f\left(x_{1}, \ldots, x_{n}\right)=\lambda\left(x_{1}, \ldots, x_{n}\right) \omega\left(x_{1}, \ldots, x_{n}\right) \prod_{c \in C}\left(\mu\left(x_{1}, \ldots, x_{n}\right)-c\right) .
$$

Then $\operatorname{deg} f=\sum_{i=1}^{n}\left(k_{i}-1\right)$, and the coefficient of $\prod_{i=1}^{n} x_{i}^{k_{i}-1}$ in $f$ is nonzero. By Theorem 1.2 of [A1], there are $a_{1} \in A_{1}, \ldots, a_{n} \in A_{n}$ such that $f\left(a_{1}, \ldots, a_{n}\right) \neq 0$. On the other hand, by the very definition of $C$, $f\left(a_{1}, \ldots, a_{n}\right)=0$ for all $a_{1} \in A_{1}, \ldots, a_{n} \in A_{n}$. So we get a contradiction.

Proposition 2.2. Let $k, m, n$ be integers with $m \geq 0, n>1$ and $k>$ $m(n-1)$. Then the coefficient of $x_{1}^{k-1} \ldots x_{n}^{k-1}$ in

$$
\prod_{1 \leq i<j \leq n}\left(x_{i}-x_{j}\right)^{2 m}\left(x_{1}+\ldots+x_{n}\right)^{n(k+m-m n-1)}
$$

coincides with

$$
(-1)^{m n(n-1) / 2} \frac{((k+m-m n-1) n) !}{(m !)^{n}} \prod_{j=1}^{n} \frac{(j m) !}{(k-1-(j-1) m) !} .
$$

To prove this proposition is the main difficulty in our paper; the proof will be presented in the next section.

Now we deduce Theorem 1.1 from Propositions 2.1 and 2.2.

Proof of Theorem 1.1. As $|F| \geq p(F)>m n \geq m$, we can extend each $S_{i j}$ $(i \neq j)$ to a subset of $F$ with cardinality $m$. Without any loss of generality, we may assume that all the $S_{i j}$ have cardinality $m$.

Let $l=k+m-m n-1$. The case $l<0$ or $n=1$ is trivial. Below we handle the case $l \geq 0$ and $n \geq 2$.

Suppose on the contrary that $|C| \leq \ln$. Put

$$
\begin{aligned}
& \lambda\left(x_{1}, \ldots, x_{n}\right)=\prod_{1 \leq i<j \leq n} \prod_{c_{i j} \in S_{i j}}\left(x_{i}-x_{j}-c_{i j}\right) \prod_{c_{j i} \in S_{j i}}\left(x_{i}-x_{j}+c_{j i}\right), \\
& \mu\left(x_{1}, \ldots, x_{n}\right)=x_{1}+\ldots+x_{n}, \\
& \omega\left(x_{1}, \ldots, x_{n}\right)=\left(x_{1}+\ldots+x_{n}\right)^{l n-|C|} .
\end{aligned}
$$

Then (2.1) holds. For

$$
f\left(x_{1}, \ldots, x_{n}\right)=\lambda\left(x_{1}, \ldots, x_{n}\right) \omega\left(x_{1}, \ldots, x_{n}\right) \mu\left(x_{1}, \ldots, x_{n}\right)^{|C|},
$$


the total degree is $m n(n-1)+\ln =n(k-1)=\sum_{i=1}^{n}\left(\left|A_{i}\right|-1\right)$ and the coefficient of $x_{1}^{k-1} \ldots x_{n}^{k-1}$ in $f\left(x_{1}, \ldots, x_{n}\right)$ is the same as that in

$$
\prod_{1 \leq i<j \leq n}\left(x_{i}-x_{j}\right)^{2 m}\left(x_{1}+\ldots+x_{n}\right)^{l n} \in F\left[x_{1}, \ldots, x_{n}\right] .
$$

By Proposition 2.2, the coefficient of $x_{1}^{k-1} \ldots x_{n}^{k-1}$ should be he where $e$ is the (multiplicative) identity of $F$ and

$$
h=(-1)^{m n(n-1) / 2} \frac{(l n) !}{(m !)^{n}} \prod_{j=1}^{n} \frac{(j m) !}{(k-1-(j-1) m) !} \in \mathbb{Z} \backslash\{0\} .
$$

In view of Proposition 2.1, we should have $h e=0$. So, $p$ is a prime dividing $h$. Since $p$ is greater than $m n$ and $l n$, we have $h \not \equiv 0(\bmod p)$ and a contradiction follows.

3. Proof of Proposition 2.2. For $k=0,1,2, \ldots$ we let

$$
(x)_{k}=\prod_{j \in[0, k)}(x-j) .
$$

(The empty product is regarded as 1.) For $f\left(x_{1}, \ldots, x_{n}\right) \in \mathbb{Q}\left[x_{1}, \ldots, x_{n}\right]$, by coeff $\left[x_{1}^{i_{1}} \ldots x_{n}^{i_{n}}\right]$ in $f\left(x_{1}, \ldots, x_{n}\right)$ we mean the coefficient of the monomial $x_{1}^{i_{1}} \ldots x_{n}^{i_{n}}$ in the polynomial $f\left(x_{1}, \ldots, x_{n}\right)$.

Let $m \geq 0$ and $n>1$ be integers. Write

$$
f_{m}\left(x_{1}, \ldots, x_{n}\right)=\prod_{1 \leq i<j \leq n}\left(x_{i}-x_{j}\right)^{2 m}=\sum_{j_{1}, \ldots, j_{n}} f_{j_{1}, \ldots, j_{n}}^{(m)} x_{1}^{j_{1}} \ldots x_{n}^{j_{n}} .
$$

For any integer $k>m(n-1)$, clearly coeff $\left[x_{1}^{k-1} \ldots x_{n}^{k-1}\right]$ in $f_{m}\left(x_{1}, \ldots, x_{n}\right)\left(x_{1}+\ldots+x_{n}\right)^{n(k-1-m(n-1))}$

$$
\begin{aligned}
& =\sum_{\begin{array}{c}
j_{1}, \ldots, j_{n} \in[0, k) \\
j_{1}+\ldots+j_{n}=m n(n-1)
\end{array}} f_{j_{1}, \ldots, j_{n}}^{(m)} \frac{((k+m-m n-1) n) !}{\left(k-1-j_{1}\right) ! \ldots\left(k-1-j_{n}\right) !} \\
& =\frac{((k+m-m n-1) n) !}{((k-1) !)^{n}} \sum_{\substack{j_{1}, \ldots, j_{n} \in[0, k) \\
j_{1}+\ldots+j_{n}=m n(n-1)}} f_{j_{1}, \ldots, j_{n}}^{(m)}(k-1)_{j_{1}} \ldots(k-1)_{j_{n}} \\
& =\frac{((k+m-m n-1) n) !}{((k-1) !)^{n}} \mathcal{L}\left(f_{m}\right)(k-1),
\end{aligned}
$$

where $\mathcal{L}: \mathbb{Q}\left[x_{1}, \ldots, x_{n}\right] \rightarrow \mathbb{Q}[x]$ is the linear operator given by

$$
\mathcal{L}\left(x_{1}^{j_{1}} \ldots x_{n}^{j_{n}}\right)=(x)_{j_{1}} \ldots(x)_{j_{n}} .
$$

Thus the main problem is to determine $\mathcal{L}\left(f_{m}\right)$. 
Lemma 3.1. Let $m$ be any positive integer. Then

$$
(x)_{0}(x)_{m} \ldots(x)_{(n-1) m} \mid \mathcal{L}\left(f_{m}\right) .
$$

Proof. Observe that

$$
(x)_{0}(x)_{m} \ldots(x)_{(n-1) m}=\prod_{q=0}^{n-1} \prod_{r=0}^{m-1}(x-(q m+r))^{n-1-q}
$$

because $\{j \in[0, n): j m-1 \geq q m+r\}=[q+1, n)$ has cardinality $n-1-q$. So it suffices to show that $(x-l)^{n-1-[l / m]} \mid \mathcal{L}\left(f_{m}\right)$ for any $l=0,1, \ldots, m n-1$.

Let $j_{1}, \ldots, j_{n}$ be nonnegative integers with $f_{j_{1}, \ldots, j_{n}}^{(m)} \neq 0$. In order to prove that $\mathcal{L}\left(x_{1}^{j_{1}} \ldots x_{n}^{j_{n}}\right)=(x)_{j_{1}} \ldots(x)_{j_{n}}$ is divisible by $(x-l)^{n-1-[l / m]}$, we only need to show that

$$
\left|\left\{1 \leq i \leq n: j_{i}>l\right\}\right| \geq n-1-\left[\frac{l}{m}\right] \text {, i.e. }\left|\left\{1 \leq i \leq n: j_{i} \leq l\right\}\right| \leq 1+\left[\frac{l}{m}\right] .
$$

Let $I=\left\{1 \leq i \leq n: j_{i} \leq l\right\} \neq \emptyset$. The polynomial $\prod_{i, j \in I, i<j}\left(x_{i}-x_{j}\right)^{2 m}$ divides $f_{m}\left(x_{1}, \ldots, x_{n}\right)$ and each monomial in it has degree $2 m\left(\begin{array}{c}|I| \\ 2\end{array}\right)=m|I|(|I|-1)$. Since $f_{j_{1}, \ldots, j_{n}}^{(m)} \neq 0$, we have $\sum_{i \in I} j_{i} \geq m|I|(|I|-1)$ and hence $l \geq j_{i} \geq$ $m(|I|-1)$ for some $i \in I$. Therefore $|I| \leq 1+[l / m]$. This concludes the proof.

Lemma 3.2. Let $g\left(x_{1}, \ldots, x_{n}\right) \in \mathbb{Q}\left[x_{1}, \ldots, x_{n}\right]$ and $1 \leq s<t \leq n$. Then

$$
\begin{aligned}
\mathcal{L}\left(\left(x_{s}-x_{t}\right) g\left(x_{1}, \ldots, x_{n}\right)\right) & \\
& =\mathcal{L}\left(x_{t} \frac{\partial g\left(x_{1}, \ldots, x_{n}\right)}{\partial x_{t}}\right)-\mathcal{L}\left(x_{s} \frac{\partial g\left(x_{1}, \ldots, x_{n}\right)}{\partial x_{s}}\right) .
\end{aligned}
$$

Proof. For any nonnegative integers $j_{1}, \ldots, j_{n}$, we have

$$
\begin{aligned}
\mathcal{L}\left(\left(x_{s}\right.\right. & \left.\left.-x_{t}\right) x_{1}^{j_{1}} \ldots x_{n}^{j_{n}}\right) \\
& =\prod_{\substack{i=1 \\
i \neq s, t}}^{n}(x)_{j_{i}} \cdot\left((x)_{j_{s}+1}(x)_{j_{t}}-(x)_{j_{s}}(x)_{j_{t}+1}\right) \\
& =(x)_{j_{1}} \ldots(x)_{j_{n}}\left(x-j_{s}-x+j_{t}\right)=j_{t}(x)_{j_{1}} \ldots(x)_{j_{n}}-j_{s}(x)_{j_{1}} \ldots(x)_{j_{n}} \\
& =\mathcal{L}\left(x_{t} \frac{\partial\left(x_{1}^{j_{1}} \ldots x_{n}^{j_{n}}\right)}{\partial x_{t}}\right)-\mathcal{L}\left(x_{s} \frac{\partial\left(x_{1}^{j_{1}} \ldots x_{n}^{j_{n}}\right)}{\partial x_{s}}\right) .
\end{aligned}
$$

Write $g\left(x_{1}, \ldots, x_{n}\right)=\sum_{j_{1}, \ldots, j_{n}} g_{j_{1}, \ldots, j_{n}} x_{1}^{j_{1}} \ldots x_{n}^{j_{n}}$ where $g_{j_{1}, \ldots, j_{n}} \in \mathbb{Q}$. Then, by the above, 


$$
\begin{aligned}
\mathcal{L}\left(\left(x_{s}\right.\right. & \left.\left.-x_{t}\right) g\left(x_{1}, \ldots, x_{n}\right)\right) \\
& =\sum_{j_{1}, \ldots, j_{n}} g_{j_{1}, \ldots, j_{n}} \mathcal{L}\left(\left(x_{s}-x_{t}\right) x_{1}^{j_{1}} \ldots x_{n}^{j_{n}}\right) \\
& =\mathcal{L}\left(\sum g_{j_{1}, \ldots, j_{n}} x_{t} \frac{\partial\left(x_{1}^{j_{1}} \ldots x_{n}^{j_{n}}\right)}{\partial x_{t}}\right)-\mathcal{L}\left(\sum g_{j_{1}, \ldots, j_{n}} x_{s} \frac{\partial\left(x_{1}^{j_{1}} \ldots x_{n}^{j_{n}}\right)}{\partial x_{s}}\right) \\
& =\mathcal{L}\left(x_{t} \frac{\partial g\left(x_{1}, \ldots, x_{n}\right)}{\partial x_{t}}\right)-\mathcal{L}\left(x_{s} \frac{\partial g\left(x_{1}, \ldots, x_{n}\right)}{\partial x_{s}}\right) .
\end{aligned}
$$

LEMMA 3.3. Let $\Delta \neq \emptyset$ be a finite multi-set whose elements are ordered pairs in the form $(i, j)$ with $1 \leq i<j \leq n$. Let $g\left(x_{1}, \ldots, x_{n}\right) \in \mathbb{Q}\left[x_{1}, \ldots, x_{n}\right]$ and $1 \leq r \leq n$. Then

$$
\begin{aligned}
\frac{\partial}{\partial x_{r}}\left(g\left(x_{1}, \ldots, x_{n}\right) \prod_{(i, j) \in \Delta}\left(x_{i}-x_{j}\right)\right) & \\
& =\sum_{(s, t) \in \Delta} \frac{g_{s, t}\left(x_{1}, \ldots, x_{n}\right)}{x_{s}-x_{t}} \prod_{(i, j) \in \Delta}\left(x_{i}-x_{j}\right)
\end{aligned}
$$

where $g_{s, t}\left(x_{1}, \ldots, x_{n}\right) \in \mathbb{Q}\left[x_{1}, \ldots, x_{n}\right]$ and $\operatorname{deg} g_{s, t} \leq \operatorname{deg} g$.

Proof. Let $(u, v)$ be any element of $\Delta$. Then

$$
\begin{aligned}
\frac{\partial}{\partial x_{r}}\left(g\left(x_{1}, \ldots, x_{n}\right) \prod_{(i, j) \in \Delta}\left(x_{i}-x_{j}\right)\right) \\
=\frac{\partial g\left(x_{1}, \ldots, x_{n}\right)}{\partial x_{r}} \prod_{(i, j) \in \Delta}\left(x_{i}-x_{j}\right)+g\left(x_{1}, \ldots, x_{n}\right) \frac{\partial}{\partial x_{r}} \prod_{(i, j) \in \Delta}\left(x_{i}-x_{j}\right) \\
=\left(\frac{\partial g\left(x_{1}, \ldots, x_{n}\right)}{\partial x_{r}}\left(x_{u}-x_{v}\right)\right) \frac{\prod_{(i, j) \in \Delta}\left(x_{i}-x_{j}\right)}{x_{u}-x_{v}} \\
\quad+g\left(x_{1}, \ldots, x_{n}\right) \sum_{(s, t) \in \Delta} \frac{\partial\left(x_{s}-x_{t}\right)}{\partial x_{r}} \cdot \frac{\prod_{(i, j) \in \Delta}\left(x_{i}-x_{j}\right)}{x_{s}-x_{t}} .
\end{aligned}
$$

Clearly $\operatorname{deg} g$ is not less than the degrees of those $g\left(x_{1}, \ldots, x_{n}\right) \frac{\partial\left(x_{s}-x_{t}\right)}{\partial x_{r}}$ (where $(s, t) \in \Delta)$ and $\frac{\partial g\left(x_{1}, \ldots, x_{n}\right)}{\partial x_{r}}\left(x_{u}-x_{v}\right)$. So the desired result follows.

Combining Lemmas 3.2 and 3.3 we have

LEMMA 3.4. Let $m$ be a nonnegative integer and $\Delta$ a multi-set with elements in the form $(i, j)(1 \leq i<j \leq n)$ and $|\Delta|$ equal to $2 m$. Then for any $g\left(x_{1}, \ldots, x_{n}\right) \in \mathbb{Q}\left[x_{1}, \ldots, x_{n}\right]$ we have

$$
\operatorname{deg} \mathcal{L}\left(g\left(x_{1}, \ldots, x_{n}\right) \prod_{(i, j) \in \Delta}\left(x_{i}-x_{j}\right)\right) \leq \operatorname{deg} g+m
$$


Proof. We use induction on $m$. The case $m=0$ is trivial, so we proceed to the induction step.

Assume $m \in \mathbb{Z}^{+}$. Let $(s, t)$ be any element in $\Delta$ and $\Delta^{\prime}$ denote the multi-set $\Delta$ with one $(s, t)$ omitted. By Lemmas 3.2 and 3.3,

$$
\begin{aligned}
\mathcal{L}\left(g\left(x_{1}, \ldots, x_{n}\right) \prod_{(i, j) \in \Delta}\right. & \left.\left(x_{i}-x_{j}\right)\right) \\
= & \mathcal{L}\left(x_{t} \frac{\partial\left(g\left(x_{1}, \ldots, x_{n}\right) \prod_{(i, j) \in \Delta^{\prime}}\left(x_{i}-x_{j}\right)\right)}{\partial x_{t}}\right) \\
& -\mathcal{L}\left(x_{s} \frac{\partial\left(g\left(x_{1}, \ldots, x_{n}\right) \prod_{(i, j) \in \Delta^{\prime}}\left(x_{i}-x_{j}\right)\right)}{\partial x_{s}}\right)
\end{aligned}
$$

can be written in the form

$$
\begin{aligned}
\mathcal{L}\left(\sum_{(u, v) \in \Delta^{\prime}} \frac{g_{u v}\left(x_{1}, \ldots, x_{n}\right)}{x_{u}-x_{v}} \prod_{(i, j) \in \Delta^{\prime}}\left(x_{i}-x_{j}\right)\right) \\
=\sum_{(u, v) \in \Delta^{\prime}} \mathcal{L}\left(\frac{g_{u v}\left(x_{1}, \ldots, x_{n}\right)}{x_{u}-x_{v}} \prod_{(i, j) \in \Delta^{\prime}}\left(x_{i}-x_{j}\right)\right)
\end{aligned}
$$

where $g_{u v}\left(x_{1}, \ldots, x_{n}\right) \in \mathbb{Q}\left[x_{1}, \ldots, x_{n}\right]$ and $\operatorname{deg} g_{u v} \leq \operatorname{deg} g+1$. Choose $(u, v) \in \Delta^{\prime}$ so that $\operatorname{deg} \mathcal{L}\left(\frac{g_{u v}\left(x_{1}, \ldots, x_{n}\right)}{x_{u}-x_{v}} \prod_{(i, j) \in \Delta^{\prime}}\left(x_{i}-x_{j}\right)\right)$ is maximal. Let $\Delta^{\prime \prime}$ be the multi-set $\Delta^{\prime}$ with one $(u, v)$ deleted. Then $\left|\Delta^{\prime \prime}\right|=2(m-1)$ and

$$
\begin{aligned}
\operatorname{deg} \mathcal{L}\left(g\left(x_{1}, \ldots, x_{n}\right) \prod_{(i, j) \in \Delta}\right. & \left.\left(x_{i}-x_{j}\right)\right) \\
& \leq \operatorname{deg} \mathcal{L}\left(g_{u v}\left(x_{1}, \ldots, x_{n}\right) \prod_{(i, j) \in \Delta^{\prime \prime}}\left(x_{i}-x_{j}\right)\right) .
\end{aligned}
$$

By the induction hypothesis,

$$
\operatorname{deg} \mathcal{L}\left(g_{u v}\left(x_{1}, \ldots, x_{n}\right) \prod_{(i, j) \in \Delta^{\prime \prime}}\left(x_{i}-x_{j}\right)\right) \leq \operatorname{deg} g_{u v}+(m-1) \leq \operatorname{deg} g+m .
$$

So we have (3.3).

Lemma 3.5. Let $m \geq 0$ and $n>1$ be integers. Then

$$
\begin{aligned}
& \text { coeff }\left[x_{1}^{m(n-1)} \ldots x_{n}^{m(n-1)}\right] \text { in } \prod_{1 \leq i<j \leq n}\left(x_{i}-x_{j}\right)^{2 m} \\
& =(-1)^{m n(n-1) / 2} \frac{(m n) !}{(m !)^{n}} .
\end{aligned}
$$

Proof. Let $m_{1}, \ldots, m_{n} \in \mathbb{N}$. When we expand $\prod_{1 \leq i, j \leq n, i \neq j}\left(1-x_{i} / x_{j}\right)^{m_{j}}$ as a Laurent polynomial in $x_{1}, \ldots, x_{n}$ (i.e., negative exponents allowed), the constant term is the multinomial coefficient $\left(\sum_{i=1}^{n} m_{i}\right) ! / \prod_{i=1}^{n}\left(m_{i} !\right)$. This 
result was conjectured by F. J. Dyson [D] in 1962. An elegant proof given by I. J. Good [Go] in 1970 uses the Lagrange interpolation formula. D. Zeilberger [Z] gave a combinatorial proof of Dyson's conjecture in the following equivalent form:

$$
\text { coeff } \begin{aligned}
{\left[x_{1}^{m_{1}(n-1)} \ldots x_{n}^{m_{n}(n-1)}\right] \text { in } } & \prod_{1 \leq i<j \leq n}\left(x_{i}-x_{j}\right)^{m_{i}+m_{j}} \\
& =(-1)^{\sum_{j=1}^{n}(j-1) m_{j}} \frac{\left(m_{1}+\ldots+m_{n}\right) !}{m_{1} ! \ldots m_{n} !} .
\end{aligned}
$$

Taking $m_{1}=\ldots=m_{n}=m$ in the above equality, we get (3.4).

Now we are ready to prove

Theorem 3.1. Let $f\left(x_{1}, \ldots, x_{n}\right)=\prod_{1 \leq i<j \leq n}\left(x_{i}-x_{j}\right)^{2 m}$ where $m \in \mathbb{N}$ and $n>1$. Then

$$
\mathcal{L}(f)=(-1)^{m n(n-1) / 2} \frac{m !(2 m) ! \ldots(n m) !}{(m !)^{n}}(x)_{0}(x)_{m} \ldots(x)_{(n-1) m} .
$$

Proof. By Lemma 3.1, there exists a $g(x) \in \mathbb{Q}[x]$ such that

$$
\mathcal{L}(f)=(x)_{0}(x)_{m} \ldots(x)_{(n-1) m} g(x) .
$$

Note that $\operatorname{deg} \prod_{j=0}^{n-1}(x)_{j m}=\sum_{j=0}^{n-1} j m=m n(n-1) / 2$. By Lemma 3.4, $\operatorname{deg} \mathcal{L}(f) \leq \operatorname{deg} 1+m\left(\begin{array}{c}n \\ 2\end{array}\right)$. So $g(x)$ is a constant $c \in \mathbb{Q}$. As we mentioned at the beginning of this section,

$$
\begin{aligned}
\text { coeff }\left[x_{1}^{m n-m} \ldots x_{n}^{m n-m}\right] \text { in } & f\left(x_{1}, \ldots, x_{n}\right) \\
= & \frac{((m n-m+m-m n) n) !}{((m n-m) !)^{n}} \mathcal{L}(f)(m n-m) .
\end{aligned}
$$

In view of Lemma 3.5, we have

$$
c \prod_{j=0}^{n-1}(m n-m)_{j m}=\mathcal{L}(f)(m n-m)=((m n-m) !)^{n} \cdot(-1)^{m n(n-1) / 2} \frac{(m n) !}{(m !)^{n}},
$$

i.e.,

$$
c=(-1)^{m n(n-1) / 2} \frac{(m n) !}{(m !)^{n}} \prod_{j=0}^{n-1}(m n-m-j m) !=(-1)^{m n(n-1) / 2} \frac{\prod_{i=1}^{n}(i m) !}{(m !)^{n}} .
$$

This ends the proof.

Proof of Proposition 2.2. Let $f\left(x_{1}, \ldots, x_{n}\right)=\prod_{1 \leq i<j \leq n}\left(x_{i}-x_{j}\right)^{2 m}$. By Theorem 3.1, we have

$$
\mathcal{L}(f)(k-1)=(-1)^{m n(n-1) / 2} \frac{m !(2 m) ! \ldots(n m) !}{(m !)^{n}} \prod_{i=0}^{n-1}(k-1)_{i m} .
$$


Thus

$$
\begin{aligned}
\text { coeff }\left[x_{1}^{k-1} \ldots x_{n}^{k-1}\right] \text { in } f\left(x_{1}, \ldots, x_{n}\right)\left(x_{1}+\ldots+x_{n}\right)^{(k-1-m(n-1)) n} \\
\quad=\frac{((k+m-m n-1) n) !}{((k-1) !)^{n}} \mathcal{L}(f)(k-1) \\
=\frac{((k+m-m n-1) n) !}{((k-1) !)^{n}}(-1)^{m n(n-1) / 2} \frac{\prod_{j=1}^{n}(j m) !}{(m !)^{n}} \prod_{j=1}^{n}(k-1)_{(j-1) m} \\
=(-1)^{m n(n-1) / 2} \frac{\prod_{j=1}^{n}(j m) !}{(m !)^{n}} \cdot \frac{((k+m-m n-1) n) !}{\prod_{j=1}^{n}(k-1-(j-1) m) !} .
\end{aligned}
$$

We are done.

Acknowledgements. The authors are indebted to Prof. N. Alon for his comments and the referee for his suggestions. The work was done during the second author's visit to the Center for Combinatorics at Nankai University; he thanks Prof. William Y. C. Chen for the invitation and the Center for its support.

\section{References}

[A1] N. Alon, Combinatorial Nullstellensatz, Combin. Probab. Comput. 8 (1999), $7-29$.

[A2] -, Additive Latin transversals, Israel J. Math. 117 (2000), 125-130.

[ANR1] N. Alon, M. B. Nathanson and I. Z. Ruzsa, Adding distinct congruence classes modulo a prime, Amer. Math. Monthly 102 (1995), 250-255.

[ANR2] - - - - , The polynomial method and restricted sums of congruence classes, J. Number Theory 56 (1996), 404-417.

[DH] J. A. Dias da Silva and Y. O. Hamidoune, Cyclic space for Grassmann derivatives and additive theory, Bull. London Math. Soc. 26 (1994), 140-146.

[D] F. J. Dyson, Statistical theory of the energy levels of complex systems I, J. Math. Phys. 3 (1962), 140-156.

$[\mathrm{EH}] \quad$ P. Erdős and H. Heilbronn, On the addition of residue classes mod $p$, Acta Arith. 9 (1964), 149-159.

[Go] I. J. Good, Short proof of a conjecture of Dyson, J. Math. Phys. 11 (1970), 1884 .

[Gu] R. K. Guy, Unsolved Problems in Number Theory, 2nd ed., Springer, New York, 1994, 129-131.

[N] M. B. Nathanson, Additive Number Theory: Inverse Problems and the Geometry of Sumsets, Grad. Texts in Math. 165, Springer, New York, 1996.

[Sn] H. S. Snevily, The Cayley addition table of $\mathbb{Z}_{n}$, Amer. Math. Monthly 106 (1999), 584-585.

[Su] Z. W. Sun, Restricted sums of subsets of $\mathbb{Z}$, Acta Arith. 99 (2001), 41-60. 
[Z] D. Zeilberger, A combinatorial proof of Dyson's conjecture, Discrete Math. 41 (1982), 317-321.

Center for Combinatorics

Nankai University

Tianjin 300071, P.R. China

E-mail: hqh@public.tpt.tj.cn
Department of Mathematics Nanjing University Nanjing 210093, P.R. China E-mail: zwsun@nju.edu.cn

Received on 23.8.1999

and in revised form on 24.7.2001 